\title{
Release of Soluble Ligands for the Activating NKG2D Receptor: One More Immune Evasion Strategy Evolved by HIV-1?
}

\author{
Erica Giuliani ${ }^{1}$, Lia Vassena ${ }^{1}$, Cristina Cerboni ${ }^{2}$, and Margherita Doria ${ }^{1} *$ \\ ${ }^{1}$ Laboratory of Immunoinfectivology, Children's Hospital Bambino Gesù, IRCCS, Piazza S. Onofrio 4, \\ 00165, Rome, Italy; ${ }^{2}$ Department of Molecular Medicine, Istituto Pasteur-Fondazione Cenci Bo- \\ lognetti, "Sapienza" University of Rome, 00161, Rome, Italy
}

\begin{abstract}
Increasing lines of evidence indicate that NKG2D, an activating receptor of natural killer (NK) and $\mathrm{CD}^{+} \mathrm{T}$ cells, plays an important role in immune responses against HIV-1. Through its ability to recognize a diverse array of ligands (NKG2DLs) induced by cell 'stress' such as viral infection, NKG2D delivers activating and co-stimulatory signals resulting in cytotoxicity and release of cytokines. Therefore, HIV-1 and other viruses have evolved clever mechanisms to counteract NKG2Ddependent immune responses. While, on one hand, the HIV-1 Vpr protein up-regulates NKG2DLs ex-

Margherita Doria pression by activating the DNA damage response (DDR) pathway, other viral proteins (Nef and Vif) have developed the capacity to reduce NKG2DLs expression levels. In addition, recent evidences suggest that HIV-1-infected CD4 ${ }^{+} \mathrm{T}_{\text {cells }}$ may release NKG2DLs, particularly MICA, in soluble form, a phenomenon that has the potential to down-modulate NKG2D on circulating lymphocytes and allow evasion of NKG2D-mediated immune responses. Indeed, despite being controversial, lower NKG2D expression was found on both NK and CD8 ${ }^{+} \mathrm{T}$ cells in HIV-1-infected patients. This review discusses recent advances in the understanding of how HIV-1 affects the NKG2D/NKG2DLs system, with a special focus on virus-induced release of soluble NKG2DLs and its functional implications for the immune surveillance of the infected host.
\end{abstract}

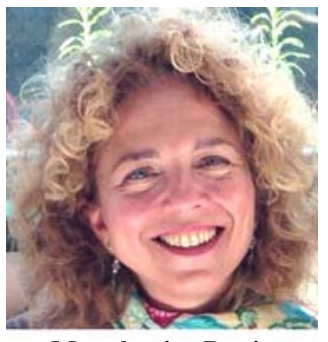

Keywords: MICA, MICB, NKG2D, shedding, ULBP1-6, HIV-1.

\section{INTRODUCTION}

The natural-killer group 2 member D (NKG2D) is one of the most studied immune receptors of the last decade. Several distinct ligands for NKG2D have been identified, all upregulated in infected, transformed, and, more in general, distressed cells. By a plethora of in vitro and in vivo studies, the crucial role of NKG2D in the immune control of viral infections and cancer has been clearly demonstrated. As discussed here, it has recently become evident that NKG2D may take an important part in the capacity of cytotoxic lymphocytes to recognize and kill HIV-1-infected $\mathrm{CD}^{+} \mathrm{T}$ cells. The present review especially examines recent results suggesting that soluble NKG2DLs are released by HIV-1-infected cells and can down-modulate NKG2D as a means of viral immune escape. The possibility that HIV-1 uses soluble NKG2DLs to subvert NKG2D-mediated immune surveillance opens the way to novel therapeutic strategies and deserves to be further explored. This discussion speculates on the latest findings and is conceived to provide ideas for future studies.

\section{AN OVERVIEW OF NKG2D AND ITS LIGANDS}

\subsection{The NKG2D Receptor}

NKG2D is a potent activating receptor constitutively expressed in humans by all natural killer (NK) cells and CD8 ${ }^{+}$

*Address correspondence to this author at the Laboratory of Immunoinfectivology, Children's Hospital Bambino Gesù, IRCCS, Piazza S. Onofrio 4, 00165, Rome, Italy; Tel: +39-06-72596823; Fax: +39-06-72596822;

E-mail:doria@uniroma2.it
$\mathrm{T}$ cells, and by subsets of $\gamma \delta \mathrm{T}$ cells, NKT cells, and $\mathrm{CD}^{+} \mathrm{T}$ cells [1]. Being widely expressed on cells of both innate and adaptive immune system, NKG2D has been involved in the regulation of immune responses during infection, neoplastic transformation as well as autoimmunity [1, 2].

NKG2D is a dimeric, type II transmembrane protein devoid of signaling properties but constitutively associated with the DAP10 adaptor molecules (four for each receptor homodimer). Ligand binding by NKG2D results in DAP10 tyrosine phosphorylation followed by recruitment and activation of the $\mathrm{p} 85$ regulatory subunit of the phosphatidylinositol 3-kinase (PI3-K) and the growth factor receptor-bound protein 2 (Grb2). The NKG2D pathway and its impact on immune cells have been intensely studied as reported in a number of reviews [1-3]. In general, the activating signal delivered by NKG2D triggers cytotoxicity and cytokine production in NK cells, thus functioning as a major activating pathway for NK-cell mediated elimination of transformed and infected cells [4]. On the other hand, NKG2D engagement on $\mathrm{CD}^{+} \mathrm{T}$ cells is not sufficient to induce cytotoxicity or cytokine production [5], with the exception of $\mathrm{CD} 8^{+} \mathrm{T}$ cells derived from tumors or inflamed tissues that respond to NKG2D triggering in the absence of other stimuli $[6,7]$. In most cases, NKG2D engagement functions as co-stimulus for $\mathrm{CD}^{+} \mathrm{T}$ cells, thus amplifying TCR-mediated activation and enhancing proliferation, cytotoxicity and cytokines release [8-10]. In addition, increasing lines of evidence indicate that NKG2D regulates the differentiation of both NK 
[11] and $\mathrm{CD} 8^{+} \mathrm{T}$ cells $[9,12]$. Finally, some studies reported NK cell-mediated killing of activated T cells via NKG2D, suggesting that the receptor exerts a negative regulation over $\mathrm{T}$-cell responses that might be relevant for the limitation of undesirable immune activation and for the maintenance of lymphocyte homeostasis $[13,14]$.

\subsection{The NKG2D Ligands (NKG2DLs)}

A very peculiar aspect that distinguishes NKG2D from other activating receptors is its capacity to bind a diverse array of ligands (generically defined as NKG2DLs) rather than one or two. The NKG2DLs are self-proteins structurally similar to the MHC class I molecules, albeit not associated with $\beta$-microglobulin or peptide, that fall in two families: the MHC-class-I-related sequence A (MICA) and B (MICB) protein family and the cytomegalovirus UL16-binding protein family consisting of six members (ULBP1-6) [15]. Despite the fact that all NKG2DLs share an MHC-class-I-like $\alpha 1 \alpha 2$ domain that binds NKG2D, they vary considerably in their sequence and receptor binding affinity, and they either are anchored to the cell membrane via a glycosylphosphatidylinositol (GPI) molecule (ULBP1-3, 6) or display transmembrane (TM) domains and cytoplasmic tails of variable length (MICA, MICB, ULBP4-5). In addition, NKG2DLs show polymorphisms in their coding sequences, particularly MICA for which more than 80 allelic variants exists, and promoters $[16,17]$. Very recently, the MICA variant most frequently expressed in Caucasians, $\mathrm{MICA}^{*} 008$, that has shorter TM and cytoplasmic regions and is synthesized as a free soluble protein, was shown to be located at the plasma membrane through the post-translational acquisition of a GPI anchor [18]. The NKG2DLs diversity should represent an advantage for the human immune system fighting against rapidly evolving viruses and cancers, as discussed in previous reviews $[19,20]$. In fact, expression of NKG2DLs is low or absent in most normal cells but it is up-regulated in virusinfected and cancer cells that, as a consequence, become exposed to NKG2D-mediated immune surveillance. The biosynthesis of NKG2DLs is practically regulated at any stage: transcription, RNA stability, translation, protein anterograde transport, protein stability, and soluble protein release from the cell. A thorough overview of this complex regulation has been recently published [20]. In brief, the transcription of $N K G 2 D L s$ genes is dependent on transcription factors such as NF- $\mathrm{BB}, \mathrm{Sp} 1$ and E2F [21-24] and on epigenetic mechanisms (e.g. histone acetylation) that regulate the accessibility of the transcription machinery to $N K G 2 D L$ promoter sequences [25]. In addition, a large body of evidence supports a key role of the DNA Damage Response (DDR) pathway, specifically of the DNA damage sensor kinases ATM (ataxia telangiectasia-mutated) and ATR (ATM and Rad3-related), in the induction of NKG2DLs expression in transformed and infected cells as well as in normal cells undergoing mitosis or other physiological processes in which DDR is activated [26]. Both enhanced transcription and mRNA stabilization have been proposed as mechanisms of NKG2DLs up-regulation resulting from activation of the DDR pathway, but the molecular details await elucidation. It is clear though, that NKG2DL mRNAs can be detected in healthy cells devoid of cellsurface ligands and that post-transcriptional mechanisms exist to prevent NKG2DL translation and expression in healthy cells. In particular, cellular microRNAs (miRNAs) that are either constitutively expressed or induced, were shown to bind NKG2DL mRNAs and repress their transcription [27-29]. On the other hand, some miRNAs targeting MICA mRNA are degraded upon lipopolysaccharide activation, indicating that NKG2DLs expression can be derepressed by infections or inflammation by elimination of coupled miRNAs [30]. At the protein stage, NKG2DLs can be negatively regulated by ubiquitination followed by rapid degradation, as demonstrated for MULT1, the murine ortholog of ULBP1 [31]. Additional mechanisms affecting NKG2DLs stability and intracellular trafficking exist, often subverted in transformed and virally infected cells (these latter are examined later in this review). Finally, the mechanisms underlying the release of soluble NKG2DLs have been intensively studied in recent years and are discussed below in a separate section.

Overall, NKG2DLs expression involves the coordinated effect of several pathways. Interestingly, there are stimuli that specifically induce only one ligand or that are effective in one cell type but not another. As a general rule, which ligand is up-regulated and to what extent reflect not only NKG2DLs diversity and polymorphism, but also the nature of the stress stimulus, the type of distressed cell and its tissue microenvironment.

\section{SHEDDING OF SOLUBLE NKG2D LIGANDS AND ITS IMPACT ON NKG2D-MEDIATED IMMUNO- SURVEILLANCE}

The capacity of cells to release soluble forms of NKG2DLs (sNKG2DLs) in the extracellular milieu was initially discovered in patients with gastrointestinal cancers [32], but later observed also in other solid tumors and in a variety of hematological malignancies and autoimmune disorders [33], as well as in physiologic contexts such as pregnancy [34] and antigen-mediated activation of $T$ cells [35]. Soluble MICA, sMICB, and sULBP2 proteins have been found in the sera of cancer patient, and basically every MIC and ULBP ligand has been detected in soluble form in culture supernatants of either cancer cells, transfected cell lines or activated $\mathrm{T}$ cells, indicating that any NKG2DL can potentially be released in the extracellular environment. The release of sNKG2DLs reduces the cell-surface ligand density and, at the same time, allows circulation of cell-free ligands with a potentially intact capacity to bind their cognate receptor. This phenomenon may thus have important functional consequences for NKG2D-mediated responses and it has been intensively investigated in the last decade.

Three distinct mechanisms for the generation and release of sNKG2DLs have been described [15, 36, 37]. First, the ectodomain of NKG2DLs can be released from the cell membrane upon cleavage by matrix metalloproteinases (MMPs), cellular endopeptidases that primarily target cell membrane proteins to regulate tissue remodeling, leukocyte migration and inflammatory processes [38]. In particular, two MMPs, MMP9 and MMP14, and some members of 'a disintegrin and metalloproteinase' (ADAM) family (i.e. ADAM9, ADAM10, ADAM15, and ADAM17), have been implicated in the cleavage of MICA, MICB, and ULBP2 on 
the basis of studies with tumor cell lines of various origin in which metalloproteinase (MP) inhibitors and siRNAmediated down-regulation of individual MPs have been used [37]. Overall, these studies also suggest that an MP is more or less effective at shedding a given NKG2DL depending on the enzyme activation status, which may vary considerably in different tumor cells.

The second mechanism involves the release of full-length ligands in exosomes, which are vesicles of endosomal origin loaded with a variety of cellular proteins and provided with immunomodulatory functions. Some studies suggested that the truncated $\mathrm{MICA}^{*} 008$ variant and ULBP3 are primarily released in exosomes, while longer MICA variants, MICB, and ULBP2, are shed by MP-mediated cleavage [39, 40]. Nonetheless, basically all MIC and ULBP proteins were detected in purified exosomes in a number of studies [41-43]. A reconciling model has been proposed for which most NKG2DLs can be released either by proteolytic shedding or via exosomes, with the first mechanism being favored when cells express activated MP enzymes and ligands undergo secondary modification that increase their susceptibility to MP-mediated cleavage (e.g. palmitoylation and conformational changes mediated by the endoplasmic reticulum disulphide isomerase protein 5 -ERP5- or heat shock $70 \mathrm{kDa}$ protein 5 -HSPA5/GRP78-) [15].

A third mechanism generating sNKG2DLs is based on alternative RNA spicing. Indeed, a splice variant transcript of ULBP4 was shown to generate a soluble protein secreted in the cell supernatant [44]. However, this is at present the only example and it is not known whether other NKG2DLs can be expressed as free soluble molecules from alternative splicing of their mRNAs.

Importantly, the accumulation of sNKG2DLs in the serum of patients with various tumors correlates with disease progression, metastasis, and poor survival [37]. Indeed, several studies have demonstrated that shedding of sNKG2DLs has detrimental effects on NKG2D-mediated immune responses. First of all, several reports showed that, by blocking MPs expression or activity, tumor cells express higher cellsurface NKG2DLs levels and become more susceptible to NKG2D-dependent lysis [45-49]. Second, a number of studies demonstrated that sNKG2DLs present in patient plasma samples or cell culture supernatant can down-regulate NKG2D on NK and T cells, resulting in receptor internalization/degradation and reduced cytotoxicity of these cells against $\mathrm{NKG}_{2} \mathrm{DL}^{+}$targets $[40,50-53]$. Moreover, in some cancer patients, elevated serum levels of sNKG2DLs, particularly sMICA, were associated with low NKG2D expression on circulating $\mathrm{NK}$ and $\mathrm{CD}^{+} \mathrm{T}$ cells $[49,53]$. Interestingly, some studies reported that NKG2DLs within exosomes are more effective at down-modulating NKG2D than their counterparts cleaved in soluble form [39, 40], possibly due to the presence of multiple molecules anchored on exosomal membranes. On the other hand, one report showed that tumor-derived $\mathrm{NKG}_{2} \mathrm{DL}^{+}$exosomes down-modulated NKG2D mainly through TGF- $\beta$ [41]. In addition, an opposite outcome was reported for $\mathrm{NKG}_{2} \mathrm{DL}^{+}$exosomes released from dendritic cells (DCs) that could directly activate NK cells ex vivo [43]. Therefore, it is evident that the effect of exosome-bound NKG2DLs on the expression and function of their cognate receptor is determined by the overall set of exosomal molecules provided with immunomodulatory activities.

Despite all evidences here described, some reports have failed to confirm that sNKG2DLs, specifically sMICB and sULBP2 derived from transfected cell lines, down-modulate NKG2D on cells exposed in vitro [46, 55]. In addition, NKG2D expression was not found consistently reduced in all patients with elevated serum levels of sMICA [56, 57]. Hence, some aspects of the immune response regulation by sNKG2DLs are still not fully elucidated. For a more comprehensive understanding, some issues should be taken into account and further investigated. First, some cell-bound NKG2DLs (i.e. MICA, MICB, and ULBP2) are apparently more effective than their secreted conterparts at decreasing NKG2D expression on in vitro exposed NK or $\mathrm{CD}^{+} \mathrm{T}$ cells $[54,55]$. Second, different ligands may vary in their capacity to engage and down-modulate $\mathrm{NKG} 2 \mathrm{D}$, as recently reported [58]. Third, secondary modifications of cell-bound, cleaved, and exosome-bound ligands that are important for their functional interaction with the receptor may not necessarily be recapitulated by in vitro experimental systems. One last very important aspect is the dual effect (NKG2D up- or downregulation) that cytokines secreted in the in vivo microenvironment may have on NKG2D expression independently of ligand engagement [59].

\section{ROLE OF THE NKG2D/NKG2DLS SYSTEM IN THE CONTROL OF VIRAL INFECTIONS}

Increased NKG2DLs expression has been reported in human cells infected with HIV-1 and several other viruses, including human cytomegalovirus (HCMV), adenovirus, Epstein-Barr virus (EBV), hepatitis $\mathrm{C}$ virus (HCV), vesicular stomatitis virus (VSV), measles virus (MV), respiratory syncytial virus (RSV), and influenza virus (FluA) [60-67]. At present, how viruses induce NKG2DLs is poorly understood. In principle, viral infection can up-regulate NKG2DLs transcription indirectly by triggering stress and signaling pathways that support activation of NF- $\mathrm{B}$ and other transcription factors implicated in ligand gene expression. These connections, however, have not been experimentally proven, as yet. Notably, some components, if not all, of the DDR pathway that controls NKG2DLs expression, are generally activated during infection as part of the host cell response against viral genome integrity and replication [68]. On the other hand, to favor their own replication, viruses have evolved the capacity to regulate the DDR pathway by either stimulating or inhibiting some of its components $[69,70]$. Actually, a link between NKG2DLs up-regulation and DDR pathway activation has been clearly demonstrated only for HIV-1 (described later in details), but one may speculate that this link will be extended to other viruses by future studies. With the mounting evidence that viral infection up-regulates ligands for an activating receptor of cytotoxic lymphocytes, it is expected that viruses have evolved means to prevent expression of these ligands at post-transcriptional levels. Indeed, this seems to be the case for some viruses including HIV-1. By means of bioinformatics and functional studies, the group of $\mathrm{O}$. Mandelboim has demonstrated that herpes viruses (specifically, HCMV, EBV, and Kaposi's sarcomaassociated herpesvirus or KSHV) and polioma viruses (i.e. 
$\mathrm{JC}$ and $\mathrm{BK}$ ) encode miRNAs that target the 3 ' untranslated region (3'UTR) of cellular mRNAs coding for MICB and ULBP3, respectively, thus impeding ligand translation and, as a consequence, NK-cell mediated lysis of infected cells $[61,71,72]$. This mechanism is highly specific and suggests that individual NKG2DL may have a precise role in a given virus-host cell interaction. To prevent expression of NKG2DLs, viruses use other mechanisms beyond miRNAs targeting that are based on virally encoded proteins generically called 'immunoevasins'. For example, HCMV encodes two proteins that bind and sequester in the endoplasmic reticulum (ER)/Golgi compartments distinct NKG2DLs: UL16, that targets MICB, and ULBP1, 2, 6, and UL142, that targets both MICA (but not MICA*008) and ULBP3 [7376]. Analogously, the adenovirus E3/19K protein sequesters intracellularly newly synthesized MICA and MICB molecules [77]. In addition, the U21 protein encoded by the human herpes virus-7 (HHV-7) relocalize ULBP1 to lysosomal compartments [78]. Finally, the K5 protein of KSHV promotes intracellular retention of MICA upon ubiquitination of its cytoplasmic tail [79]. Importantly, in all these instances, the activity of viral immunoevasins resulted in impairment of NKG2DLs cell-surface expression and protection from NKG2D-mediated killing by NK cells. One last example is HIV-1, which was recently found to encode two immunoevasins acting at different steps of NKG2DLs biogenesis (see below).

\section{IMPACT OF HIV-1 ON NKG2DLS EXPRESSION}

\subsection{NKG2DLs Up-Regulation by HIV-1}

The very first evidence that HIV-1 up-regulates NKG2DLs was obtained in our laboratory [62]. Specifically, by flow cytometric analysis, we observed that the cellsurface expression of ULBP1 and ULBP2, but not MICA, was induced on HIV-infected primary $\mathrm{CD}^{+} \mathrm{T}$ cells at day 5 post-infection with the HIV-1 NL4-3 strain and cell activation (that is necessary to allow viral replication). These results were confirmed by others and linked to the capacity of NK cells to kill HIV-infected T cell targets through NKG2D activation [80]. An important study by Fogli et al. showed that ex vivo expanded $\mathrm{HIV}$-infected $\mathrm{CD} 4^{+} \mathrm{T}$ cells derived from $\mathrm{HIV}^{+}$patients, express ULBP2 and low levels of ULBP1 and ULBP3, hence are susceptible to lysis by autologous NK cells in a manner that mainly depends on NKG2D activation [81]. More recently, we found that HIV-1 infection also up-modulates MICA and MICB expression soon after stimulation (day 3 post-activation) of resting $\mathrm{CD}^{+} \mathrm{T}$ lymphocytes previously exposed to the virus if compared with non-exposed control cells [82]. Of note, T cell activation itself, a prerequisite for HIV-1 replication, induces NKG2DLs transient expression with a dual kinetics: the MIC proteins are up-regulated first, with a pick at day 3 postactivation, while ULBPs are induced later and reach a maximum at day 5 [14]. The fast kinetics of MICs upregulation explains why the effect of HIV-1 on these ligands was not observed when only late post-activation time points (5-7 days) were investigated [62, 80, 81, 83].

One of the accessory proteins of HIV-1, Vpr (viral protein $\mathrm{R}$ ), induces a $\mathrm{G}_{2}$ cell-cycle arrest through a multistep process that involves binding to the cellular cullin-ring E3 ubiquitin ligase (DDB1-CUL4A) and induction of the DDR pathway via ATR activation [84]. Therefore, based on the fact that NKG2DLs can be induced upon activation of the DDR pathway, two groups independently investigated whether the HIV-1 Vpr protein, expressed alone or in the context of virally infected cells, was capable of up-regulating NKG2DLs $[85,86]$. By comparing primary $\mathrm{CD}^{+}{ }^{+} \mathrm{T}$ cells infected with wild type (wt) vs Vpr-deficient HIV-1 ( $\Delta \mathrm{Vpr})$, both studies clearly demonstrated that HIV-1-induced upregulation of NKG2DL, specifically ULBP2, was dependent on the presence of the Vpr protein. Apparently, only ULBP2 was strongly up-regulated on the membrane of infected cells, although experimental evidences indicate that $\mathrm{Vpr}$ increases various NKG2DLs at the transcriptional levels (i.e. ULBP1 and ULBP2, but also, to a much lesser extent, ULBP3, MICA, and MICB). Thus, it is possible that these studies missed the precise time points at which NKG2DLs other than ULBP2 are up-modulated on the membrane of infected $T$ cells. The effect of Vpr on NKG2DL expression was maintained when the protein was expressed individually but it was impaired by mutations abrogating the capacity to recruit the E3 ubiquitin ligase complex and to activate the ATR kinase or when the activity of cellular ATR was inhibited. Taken together, these data indicate that $\mathrm{Vpr}$ up-regulates NKG2DLs through recruitment of the E3 ubiquitin ligase complex and ATR-mediated activation of the DDR pathway (Fig. 1). The same molecular interactions are also required for $V p r$ capacity to arrest infected cells in $G_{2}$, a cell-cycle phase enabling efficient viral replication, therefore NKG2DLs up-regulation might be secondary to the $\mathrm{G}_{2}$ arrest. Work performed in E. Cohen laboratory also demonstrated that Vpr associated with non-infectious HIV-1 particles as well as extracellular soluble $\mathrm{Vpr}$, can up-regulate ULBP2 upon transduction of bystander T cells [86, 87], a phenomenon that should predictably contribute to $\mathrm{CD} 4^{+} \mathrm{T}$ cell loss by NKG2D-mediated cytotoxic responses against bystander uninfected $\mathrm{ULBP}^{+}$cells. The fine mechanistic details of Vpr-induced up-regulation of NKG2DLs yet need to be elucidated. In this context, it would be interesting to investigate the role of NF- $\mathrm{KB}$ and Sp1 transcription factors, which have been implicated in both $N K G 2 D L$ genes expression [21,22] and in Vpr-induced transactivation of the HIV1 long terminal promoter region [88].

Finally, it would be important to investigate whether, during HIV-1 infection, other factors besides Vpr can contribute to DDR triggering and, consequently, to NKG2DLs up-regulation. One of these factors may be the DNA damage sensor kinase ATM, which becomes activated during HIV-1 DNA integration (Fig. 1) [89]. Moreover, a study by Norman et al. showed that the apolipoprotein B-editing complex $3 \mathrm{G}$ (APOBEC $3 \mathrm{G}$ or $\mathrm{A} 3 \mathrm{G}$ ), a cellular antiviral factor that inhibits HIV-1 replication by deaminating cytidine residues to uridine, activates the DDR pathway resulting in increased NKG2DLs expression and sensitization of infected $T$ cells to NK-cell mediated killing (Fig. 1) [90]. This interesting study suggests a novel anti-HIV-1 function for A3G beyond direct damaging of the viral genome, which consists in the initiation of a cellular 'suicide mission' meant to clear infected cells through lysis by NK cells. 


\subsection{NKG2DLs Down-Regulation by HIV-1}

As a countermeasure for NKG2DLs up-regulation, HIV1 has developed means to inhibit cell-surface ligand expression.

In particular, work from our group has shown that the viral Nef protein has the ability to lower the cell-surface expression of MICA, ULBP1, and ULBP2, with a stronger effect on the latter molecule, and to reduce NKG2Ddependent killing by NK cells (Fig. 1) [62]. This function of Nef was active in the context of viral replication, because NKG2DLs up-regulation was further enhanced in primary $\mathrm{CD}^{+} \mathrm{T}$ cells infected with a Nef-deficient HIV-1 if compared to the expression of cells infected with wt virus, as observed by our own and other groups $[62,82,90]$. The HIV-1 Nef protein has the capacity to interfere with the cellsurface expression of several membrane proteins including CD4 and CXCR4 virus receptors, antigen presenting molecules (e.g. MHC-I), and co-stimulatory molecules (e.g. CD28) with important functional consequences for viral pathogenesis and in vivo spread [91]. To do so, Nef acts as a multifunctional adaptor that misdirects cellular proteins away from the plasma membrane through increased internalization, retention within intracellular compartments, and, in some cases, re-routing to lysosomes for degradation [92].
Mutagenesis-based studies have established that the capacities of Nef to interact with cellular cofactors and to downregulate CD4 and MHC-I require specific amino acid residues and are genetically separable. However, none of several Nef mutants tested (e.g. defective for the down-modulation of CD4 and/or MHC-I and for association with SH3 domains) lost the capacity to reduce NKG2DLs cell-surface expression [62], suggesting that Nef uses a distinct, not yet identified mechanism to target NKG2DLs. Moreover, Nef might use different mechanisms to down-regulate each individual NKG2DL, since all Nef variants analyzed had a stronger activity on ULBP2 if compared to MICA and ULBP1, and patient-derived Nef variants were active on MICA and ULBP2, but not on ULBP1. A larger screening of mutated and patient-derived Nef proteins for their activity on different NKG2DLs and a thorough analysis of the molecular mechanisms involved are clearly needed.

A second HIV-1 protein that contrasts NKG2DLs expression is the viral infectivity factor (Vif). Vif interacts with a Cullin 5-ElonginB/C E3 ligase to target cellular A3G for degradation, hence overcoming antiviral host restriction imposed by A3G. By decreasing A3G levels, Vif also counteracts the enhancement of NKG2DLs expression due to A3Gmediated activation of the DDR pathway, as demonstrated by Norman and colleagues [90]. By comparing $\mathrm{T}$ cells in-

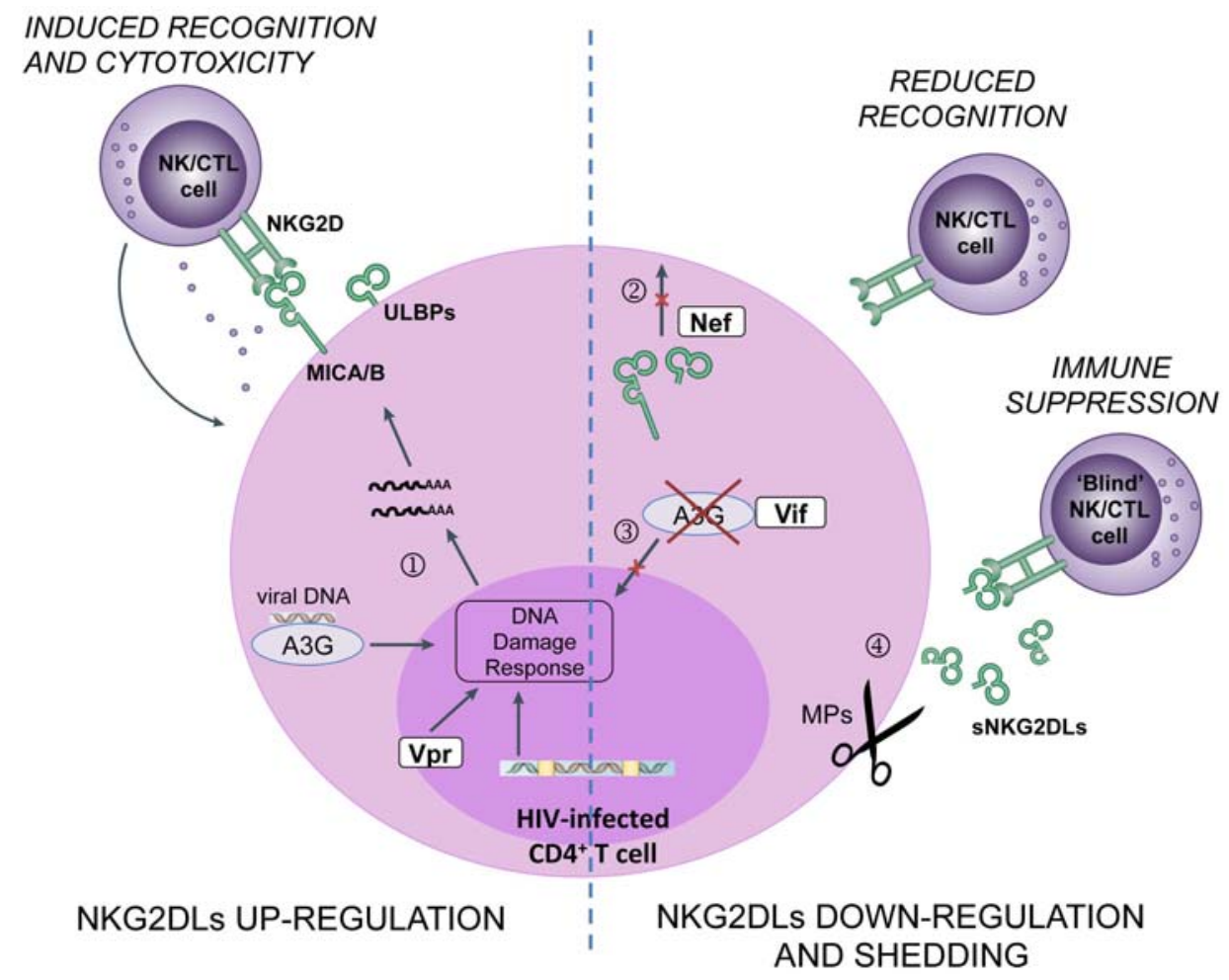

Fig. (1). A schematic model of the NKG2D/NKG2DLs system in the context of HIV-1 infection. (Left) In CD4 ${ }^{+} \mathrm{T}$ lymphocytes infected with HIV-1, the DNA damage response pathway (DDR) is triggered by the Vpr viral protein, by activated APOBEC3G (A3G), and by viral DNA integration, resulting in increased NKG2DLs mRNA levels and cell-surface NKG2DLs expression (1). Consequently, infected cells become susceptible to NKG2D-mediated recognition and killing by cytotoxic lymphocytes (NK cells and CD $8^{+}$cytotoxic T lymphocytes or CTLs). (Right) The cell-surface NKG2DLs expression is restrained by two HIV-1 immunoevasins, Nef (2) and Vif (3), this latter by inducing A3G degradation and limiting DDR activation. In addition, activated cellular metalloproteinases (MPs) cleave NKG2DLs so that their ectodomain is released in soluble form (sNKG2DLs) in the extracellular milieu (4). Lower NKG2DLs expression results in reduced activation of NKG2D ${ }^{+}$cytotoxic lymphocytes. Circulating sNKG2DLs can bind to their cognate receptor causing its systemic down-regulation on NK cells and CTLs and, thus, compromising the immunological competence of HIV-1-infected patients. 
fected with HIV-1 wt or defective for the expression of Vif, Vpr, or both viral proteins, Norman et al. demonstrated that, similarly to Nef, Vif prevented the optimal Vpr-mediated upregulation of NKG2DLs. By means of Vif mutants, the authors also showed that the negative effect of Vif on NKG2DLs expression specifically depends on its capacity to bind and degrade $\mathrm{A} 3 \mathrm{G}$.

In sum, the capacities of Nef and Vif to restrain NKG2DLs expression render HIV-1-infected cells less susceptible to NKG2D-dependent immune recognition. These activities are not very efficient, because higher NKG2DL levels are found on $\mathrm{CD} 4^{+} \mathrm{T}$ cells infected with wt virus (i.e. expressing both Nef and Vif) if compared to non-infected cells $[62,90]$. However, reduced NKG2DL expression sums up with other immune evasion strategies that HIV-1 has evolved to escape from recognition and killing by NK cells (e.g. down-regulation of NTB-A, NKp44L and PVR) [93-95] and $\mathrm{CD}^{+} \mathrm{T}$ cells (e.g. MHC-I down-regulation by Nef) [96]. It is reasonable to envision that, all together, these escape mechanisms will result in a less efficient recognition and destruction of virus-producing cells by cytotoxic lymphocytes and, consequently, in a significant increase of HIV-1 replication and spread in the infected host.

\subsection{Soluble NKG2DLs Shedding by HIV-1}

The eventuality that viral infection, analogously to tumorigenic transformation, could determine the release of sNKG2DL was investigated in a study on HIV-1-infected patients [83]. Nolting et al., found higher levels of sMICA in the plasma of patients with chronic untreated HIV-1 infection if compared to healthy HIV-negative individuals or HIV-1-infected subjects who spontaneously controlled viral replication (controllers). Conversely, aviremic HIV-1infected patients treated with highly active antiretroviral therapy (HAART) had an intermediate phenotype, displaying levels of sMICA that were significantly higher if confronted with controllers but not with HIV-negative controls. Increased sMICA in untreated HIV-1-infected patients was accompanied by an up-regulation of some MMPs (MMP2 and MMP7, but not MMP1, MMP9 and the ERP5 enzyme) in $\mathrm{CD}^{+} \mathrm{T}$ lymphocytes, supporting the hypothesis that circulating sMICA was released via MMP-mediated shedding from $\mathrm{CD}^{+} \mathrm{T}$ cells exposed to HIV-1. In line with this model, in vitro infected $\mathrm{CD}^{+} \mathrm{T}$ cells expressed higher amounts of MICA mRNA and also, when treated with MMP-inhibitors, elevated cell-surface MICA levels. Moreover, in all HIV-infected patients, both HAART-treated and, even more, untreated subjects, with the exception of controllers, NK cells had a reduced NKG2D expression and defective NKG2D-dependent functions (degranulation and activation) compared with controls. The authors of this study conclude that, like some tumors, HIV-1 may suppress NKG2Ddependent functions of NK cells by enhancing MMPmediated secretion of SMICA.

In our laboratory, we further investigated this aspect by means of in vitro infected $\mathrm{T}$ cells and patient-derived samples. First, we demonstrated that HIV-1 infection of primary $\mathrm{CD}^{+} \mathrm{T}$ cells results in increased expression and release in the culture medium of the three NKG2DLs tested, namely MICA, MICB, and ULBP2 [82]. We also showed that incu- bation with a broad spectrum MMP-inhibitor resulted in a further increase of ligands on the membrane of HIV-1infected $\mathrm{T}$ cells and decreased ligand accumulation in the culture medium, but had irrelevant effects in control noninfected $\mathrm{T}$ cell cultures. Then, by treating plasma samples with detergent in order to release NKG2DLs from macrocomplexes, we disclosed the presence of sMICA, sMICB, and SULBP2 in the plasma of several healthy individuals, in line with previously reported evidence that ligand expression and shedding can occur in normal cells undergoing physiologic processes, such as activation, proliferation, and differentiation [35, 97]. Notwithstanding, untreated patients with chronic HIV-1 infection displayed increased plasma levels of sMICA (and a similar, not statistically significant trend for sULBP2) and reduced NKG2D expression on circulating NK and $\mathrm{CD}^{+} \mathrm{T}$ cells if compared to HAART-treated patients or healthy donors. Intrapatient analysis before and after HAART treatment, clearly demonstrated that effective antiviral therapy reduced plasma sMICA and sULBP2 levels and strongly increased NKG2D expression on both $\mathrm{NK}$ and $\mathrm{CD}^{+} \mathrm{T}$ cells in HIV-1-infected patients. These results suggest that, in patients with chronic HIV-1 infection, the accumulation of sNKG2DLs released by infected $\mathrm{CD}^{+} \mathrm{T}$ cells causes systemic down-regulation of the cognate receptor on effector cells, but HAART has the potential to avoid such immune dysfunction. Finally, we demonstrated that, indeed, sNKG2DLs in patients' plasma had the capacity to downregulate $\mathrm{NKG} 2 \mathrm{D}$ on $\mathrm{NK}$ and $\mathrm{CD}^{+} \mathrm{T}$ cells and to impair NKG2D-mediated cytotoxicity of NK cells. This last set of experiments was performed by incubating peripheral blood mononuclear cells (PBMCs) of HIV-negative donors with the plasma of untreated HIV-1-infected patients, immunodepleted or not of sMICA or sULBP2, or with the plasma of healthy individuals devoid of sNKG2DLs. However, for some plasma/NK cells combinations investigated we were not able to ascribe to sMICs and sULBP2 the observed down-regulation of NKG2D or inhibition of NK cells cytotoxicity, which could be explained by the presence of other not tested NKG2DLs or distinct soluble factors regulating NKG2D.

Taken together, results from Nolting and colleagues and from our laboratory support a model in which HIV-1 induces infected $\mathrm{CD}^{+}{ }^{\mathrm{T}}$ cells to release sNKG2DLs, at least sMICA, as a means to evade NKG2D-mediated immune recognition and, simultaneously, to 'detune' the NKG2D receptor on circulating cytotoxic lymphocytes (Fig. 1). In both studies, the release of sNKG2DLs by HIV-1 seems to rely on the function of cellular MMPs, which is in line with the established role of these enzymes in ligand shedding from various tumor cells [37] and with the reported MMP up-modulation during HIV-1 infection [98].

However, various aspects of the NKG2DLs shedding by HIV-1 are still obscure or very preliminary. Some controversial points that deserve extensive investigation are discussed here below:

i) Not all HIV-1-infected patients consistently presented high amounts of circulating sMICA or sULBP2. A simplistic explanation for the absence of sMICA or sULBP2 in the plasma of several untreated HIV-1-infected patients analyzed $[82,83,99$, and our unpublished results] can be that these 
individuals express ligand alleles not responsive to virally induced shedding. Interestingly, in a previous study it was shown that, despite the great majority of healthy individuals express MICA on the surface of activated T cells, sMICA is released in only $40 \%$ to $70 \%$ of subjects, depending on the time point analyzed [35]. On the other hand, $T$ cells derived from healthy donors always released SMICB upon antigenic stimulation [35] and very high sMICB levels can be found in the plasma of some healthy individuals [82]. Therefore, the responsiveness of various ligand alleles to shedding may vary among individuals independently of viral infection. Further investigation is needed to evaluate whether and how the polymorphisms of $N K G 2 D L s$ can influence ligand expression and shedding in both healthy and HIV-infected individuals.

ii) There is no relationship between sMICA or sULBP2 levels and viral load or $\mathrm{CD}^{+} \mathrm{T}$ cell count. Despite the fact that accumulation of sMICA or sULBP2 occurred in some HIV-infected individuals, no relationship was found between the amounts of individual ligands and $\mathrm{CD}^{+} \mathrm{T}$ cell count or viral load [82]. Therefore, the accumulation of sNKG2DL could not be directly linked to viral replication, at least at the single time point analyzed.

iii) NKG2D expression levels do not show an inverse correlation with sNKG2DL amounts. The level of NKG2D expression in HIV-1-infected patients or healthy donors did not show an inverse correlation with the amount of each ligand tested (our unpublished observation), which could be due to the fact that accumulation of sNKG2DLs and sNKG2DL-induced receptor down-regulation occur transiently and follow different kinetics or, alternatively, that reduced NKG2D expression results from the combined increase of multiple ligands or other regulatory factors not yet evaluated. Adding to the overall complexity, the capacity of $\mathrm{NK}$ and $\mathrm{T}$ cells to respond to NKG2D engagement is highly variable among humans and individual NKG2D-mediated immune responses are tuned to optimal ligand doses that may vary considerably [100]. Moreover, ligand alleles or receptor haplotypes may influence the individual NKG2D responsiveness by determining qualitative effects (e.g. binding affinity). Finally, it is plausible that the concentration of sNKG2DLs in those tissues and secondary lymphoid organs where HIV-1 replication preferentially occurs could be a lot higher than that detected in the plasma.

\section{EXPRESSION AND FUNCTION OF NKG2D DUR- ING HIV-1 INFECTION}

Several ex vivo studies investigated the expression and function of the NKG2D receptor in cells derived from HIV1 -infected patients and healthy donors, often coming to discordant conclusions.

Some studies have shown that NK cells from viremic HIV-1-infected patients maintain normal NKG2D expression $[99,101-103]$ and also conserved NKG2D-mediated cytotoxicity as measured by a redirected killing assay in one report [101]. On the contrary, lower NKG2D expression on NK cells from viremic patients if compared with healthy donors was described in other studies [82, 83,104], including one in which dysfunctional receptor down-regulation in response to stimulation was observed [83]. Even more controversial is the expression of NKG2D on NK cells from HAART-treated aviremic patients, which was found either maintained [82, 101], decreased [83], or increased [105] by comparison with healthy individuals. Moreover, either normal [83], higher [105], or lower NKG2D expression [99] was described for NK cells from HIV-1 controllers as compared to healthy subjects.

Overall, the diversified pattern of NKG2D expression and function in NK cells from a given group of patients suggests that other variables beyond the clinical, virological, and immunological parameters that have been taken into consideration so far, crucially controls the receptor in these cells. It is very likely that individual variations and polymorphisms of the NKG2DL/NKG2D system may account for incongruences within the same group of patients. In this matter, longitudinal studies may possibly bring to more homogenous results. Indeed, as mentioned earlier, the analysis of the same patients before and after HAART initiation disclosed that effective viral suppression resulted in a dramatic increase in NKG2D expression on NK and $\mathrm{CD}^{+} \mathrm{T}$ cells associated with a drop of circulating sNKG2DLs [82].

As to $\mathrm{CD}^{+} \mathrm{T}$ cells, the expression and function of NKG2D on these cells in the context of HIV-1 infection remains largely unexplored. In two pilot studies, lower NKG2D expression on $\mathrm{CD}^{+} \mathrm{T}$ cells from untreated HIV-1infected patients compared to healthy donors was observed $[82,99]$. Lecuroux et al. also showed that both total and HIV-specific CD8 ${ }^{+} \mathrm{T}$ cells expressed lower NKG2D levels in different groups of HIV-infected patients (with primary infection, untreated, and HAART-treated) but not in patients with spontaneous viral suppression. These data indicate that defective NKG2D expression may contribute to impaired cytotoxic responses of $\mathrm{CD}^{+}{ }^{+} \mathrm{T}$ cells against HIV-1 as well as other pathogens. Further investigation is needed, especially since a recent study clearly underscored the importance of the NKG2D pathway in anti-HIV-1 $\mathrm{CD}^{+} \mathrm{T}$ cell responses [106]. Specifically, Zloza and colleagues showed that NKG2D co-stimulation during priming was capable of rescuing the recall capability of CD4-unhelped memory $\mathrm{CD} 8^{+} \mathrm{T}$ cells in both experimental mice models and ex vivo human cells, including $\mathrm{CD} 8^{+} \mathrm{T}$ cells derived from HIV-1-infected individuals with low $\mathrm{CD}^{+} \mathrm{T}$ cell counts. The augmented costimulation through NKG2D resulted in the suppression of the transcription factor T-box expressed in $\mathrm{T}$ cells (T-bet) and in the recovery of IL- 2 and IFN- $\gamma$ production and cytolytic responses, thus rescuing $\mathrm{CD} 8^{+} \mathrm{T}$ cell responses from chronic HIV-infected patients to resemble those associated with $\mathrm{HIV}^{+}$long term non progressor patients.

\section{THERAPEUTIC IMPLICATIONS}

Recent results here overviewed indicate that NKG2DLs shedding by HIV-1 can provoke NKG2D down-regulation and impair the responses of NK cells and, presumably, $\mathrm{CD} 8^{+}$ $\mathrm{T}$ cells, thus compromising the immunological competence of infected patients. Therefore, understanding the mechanisms through which the virus induces the release of sNKG2DLs and, subsequently, developing therapeutic strategies to suppress shedding of these molecules, may offer a substantial benefit for HIV-infected patients. 
In principle, an ideal therapeutic strategy should consist in maximizing the expression of NKG2DL on HIV-1infected cells and, at the same time, impairing ligand shedding and NKG2D down-regulation on cytotoxic lymphocytes. Several anti-cancer therapies that are currently in preclinical and clinical development, combine drugs that upregulate NKG2DLs (e.g. conventional chemotherapeutic drugs, DNA damaging agents, histone deacetylase inhibitors), with agents that induce NKG2D expression (e.g. IL-15, anti-TGF- $\beta$ monoclonal antibody) or inhibit sNKG2DLs shedding (e.g. the multi-target kinase inhibitor Sorafenib) [107]. Following an analogous combinatorial strategy, the association of agents boosting the NKG2D/NKG2DLs system with antiretroviral therapy or HIV-1-specific therapeutic vaccination, should be considered to maximize the effects of such approaches. Indeed, immunotherapy with IL-15, IL-7 and other common $\gamma$-chain signaling cytokines that upregulate NKG2D expression, is being evaluated in conjunction with HAART or HIV-1 vaccine [108]. Moreover, although still highly experimental, the use of MMP inhibitors that can block sNKG2DLs shedding has been proposed as adjuvant for anti-HIV therapy [98].

\section{CONCLUDING REMARKS AND PERSPECTIVES}

A better and more comprehensive understanding of the biochemistry and biological functions of individual NKG2DLs is clearly needed. In recent years, novel NKG2DL polymorphisms and secondary modifications have been regularly discovered and the list of stimuli that induce NKG2DL expression and of mechanisms that regulate ligand biogenesis has grown constantly. Despite intensive investigation, several aspects regarding $\mathrm{NKG} 2 \mathrm{DL}$ regulation are poorly understood, as yet, including the molecular mechanisms of ligand shedding, secretion, and exosome-mediated release, and their consequences for NKG2D function. In addition, it has become increasingly clear that the NKG2D receptor itself is under very stringent control. Indeed, the NKG2D-NKG2DL interaction has the potential to either activate or desensitize NK cells or $\mathrm{CD}^{+} \mathrm{T}$ cells, possibly depending on the nature of the ligand (i. e. genotype, isoform, secondary structure, cell/exosome-bound vs soluble) and on its relative abundance and persistence.

Along with the yet elusive aspects of the NKG2D/NKG2DLs system, clearly much work remains to be done in order to elucidate the fine mechanisms through which HIV-1 subverts this system to affect NKG2Dmediated immune surveillance. In future studies on sNKG2DLs shedding, it will be important to identify which NKG2DL genotypes are affected by the virus, also extending the analysis to ligands that have not been investigated, as yet. Furthermore, deciphering which cellular MPs mediate sNKG2DLs release by infected cells and how these enzymes are activated by the virus will certainly be of value. In fact, HIV-1 may induce MPs expression and function directly within infected cells or by the establishment of a state of chronic immune activation in the infected host. On the other hand, antiretroviral drugs can target MMPs [109], therefore it will be interesting to investigate whether low/undetectable plasma levels of sNKG2DLs and normal NKG2D expression in treated HIV-1-infected patients can be ascribed to
HAART-mediated impairment of MMPs activity and to what extent.

\section{CONFLICT OF INTEREST}

The authors confirm that this article content has no conflicts of interest.

\section{ACKNOWLEDGEMENTS}

We would like to thank a former member of the laboratory, Giulia Matusali, for contributing to this study.

This work was supported by grants of the Italian Ministry of Health, Ricerca Finalizzata and Ricerca Corrente cofunded by the Italian $5 \times 1000$ contribution to M.D and by grants of the Pasteur Institute - Cenci Bolognetti Foundation and of the "Sapienza" University of Rome to C.C.

\section{REFERENCES}

[1] Raulet DH. Roles of the NKG2D immunoreceptor and its ligands. Nat Rev Immunol 2003; 3: 781-90.

[2] Champsaur M, Lanier LL. Effect of NKG2D ligand expression on host immune responses. Immunol Rev 2010; 235: 267-85.

[3] Coudert JD, Held W. The role of the NKG2D receptor for tumor immunity. Semin Cancer Biol 2006; 16: 333-43.

[4] Bryceson YT, March ME, Ljunggren HG, Long EO. Synergy among receptors on resting NK cells for the activation of natural cytotoxicity and cytokine secretion. Blood 2006; 107: 159-66.

[5] Jamieson AM, Diefenbach A, McMahon CW, Xiong N, Carlyle JR, Raulet DH. The role of the NKG2D immunoreceptor in immune cell activation and natural killing. Immunity 2002; 17: 19-29.

[6] Maccalli C, Pende D, Castelli C, Mingari MC, Robbins PF, Parmiani G. NKG2D engagement of colorectal cancer-specific $T$ cells strengthens TCR-mediated antigen stimulation and elicits TCR independent anti-tumor activity. Eur J Immunol 2003; 33: 2033-43.

[7] Meresse B, Chen Z, Ciszewski C, et al. Coordinated induction by IL15 of a TCR-independent NKG2D signaling pathway converts CTL into lymphokine-activated killer cells in celiac disease. Immunity 2004; 21: 357-66.

[8] Groh V, Rhinehart R, Randolph-Habecker J, Topp MS, Riddell SR, Spies T. Costimulation of CD8alphabeta T cells by NKG2D via engagement by MIC induced on virus-infected cells. Nat Immunol 2001; 2: 255-60.

[9] Maasho K, Opoku-Anane J, Marusina AI, Coligan JE, Borrego F. NKG2D is a costimulatory receptor for human naive CD8+ T cells. J Immunol 2005; 174: 4480-4.

[10] Barber A, Sentman CL. NKG2D receptor regulates human effector T-cell cytokine production. Blood 2011; 117: 6571-81.

[11] Zafirova B, Mandaric S, Antulov R, et al. Altered NK cell development and enhanced NK cell-mediated resistance to mouse cytomegalovirus in NKG2D-deficient mice. Immunity 2009; 31: 27082.

[12] Wensveen FM, Lenartic M, Jelencic V, et al. NKG2D induces Mcl1 expression and mediates survival of CD8 memory $\mathrm{T}$ cell precursors via phosphatidylinositol 3-kinase. J Immunol 2013; 191: 130715 .

[13] Rabinovich BA, Li J, Shannon J, et al. Activated, but not resting, T cells can be recognized and killed by syngeneic NK cells. J Immunol 2003; 170: 3572-6.

[14] Cerboni C, Zingoni A, Cippitelli M, Piccoli M, Frati L, Santoni A Antigen-activated human $\mathrm{T}$ lymphocytes express cell-surface NKG2D ligands via an ATM/ATR-dependent mechanism and become susceptible to autologous NK- cell lysis. Blood 2007; 110: 606-15.

[15] Fernandez-Messina L, Reyburn HT, Vales-Gomez M. Human NKG2D-ligands: cell biology strategies to ensure immune recognition. Front Immunol 2012; 3: 299.

[16] Eagle RA, Traherne JA, Ashiru O, Wills MR, Trowsdale J. Regulation of NKG2D ligand gene expression. Hum Immunol 2006; 67: 159-69. 
[17] Kasahara M, Yoshida S. Immunogenetics of the NKG2D ligand gene family. Immunogenetics 2012; 64: 855-67.

[18] Ashiru O, Lopez-Cobo S, Fernandez-Messina L, et al. A GPI anchor explains the unique biological features of the common NKG2D-ligand allele MICA*008. Biochem J 2013; 454: 295-302.

[19] Eagle RA, Trowsdale J. Promiscuity and the single receptor: NKG2D. Nat Rev Immunol 2007; 7: 737-44.

[20] Raulet DH, Gasser S, Gowen BG, Deng W, Jung H. Regulation of ligands for the NKG2D activating receptor. Annu Rev Immunol 2013; 31: 413-41

[21] Molinero LL, Fuertes MB, Girart MV, et al. NF-kappa B regulates expression of the MHC class I-related chain A gene in activated T lymphocytes. J Immunol 2004; 173: 5583-90.

[22] Venkataraman GM, Suciu D, Groh V, Boss JM, Spies T. Promoter region architecture and transcriptional regulation of the genes for the MHC class I-related chain A and B ligands of NKG2D. J Immunol 2007; 178: 961-9.

[23] Jung H, Hsiung B, Pestal K, Procyk E, Raulet DH. RAE-1 ligands for the NKG2D receptor are regulated by E2F transcription factors, which control cell cycle entry. J Exp Med 2012; 209: 2409-22.

[24] Soriani A, Iannitto ML, Ricci B, et al. Reactive oxygen speciesand DNA damage response-dependent NK cell activating ligand upregulation occurs at transcriptional levels and requires the transcriptional factor E2F1. J Immunol 2014; 193: 950-60.

[25] Gonzalez S, Lopez-Soto A, Suarez-Alvarez B, Lopez-Vazquez A, Lopez-Larrea C. NKG2D ligands: key targets of the immune response. Trends Immunol 2008; 29: 397-403.

[26] Cerboni C, Fionda C, Soriani A, et al. The DNA Damage Response: A Common Pathway in the Regulation of NKG2D and DNAM-1 Ligand Expression in Normal, Infected, and Cancer Cells. Front Immunol 2014; 4: 508.

[27] Stern-Ginossar N, Gur C, Biton M, et al. Human microRNAs regulate stress-induced immune responses mediated by the receptor NKG2D. Nat Immunol 2008; 9: 1065-73.

[28] Yadav D, Ngolab J, Lim RS, Krishnamurthy S, Bui JD. Cutting edge: down-regulation of MHC class I-related chain A on tumor cells by IFN-gamma-induced microRNA. J Immunol 2009; 182: 39-43.

[29] Heinemann A, Zhao F, Pechlivanis S, et al. Tumor suppressive microRNAs miR-34a/c control cancer cell expression of ULBP2, a stress-induced ligand of the natural killer cell receptor NKG2D. Cancer Res 2012; 72: 460-71.

[30] Eissmann P, Evans JH, Mehrabi M, Rose EL, Nedvetzki S, Davis DM. Multiple mechanisms downstream of TLR-4 stimulation allow expression of NKG2D ligands to facilitate macrophage/NK cell crosstalk. J Immunol 2010; 184: 6901-9.

[31] Nice TJ, Coscoy L, Raulet DH. Posttranslational regulation of the NKG2D ligand Mult1 in response to cell stress. J Exp Med 2009; 206: 287-8.

[32] Salih HR, Rammensee HG, Steinle A. Cutting edge: downregulation of MICA on human tumors by proteolytic shedding. J Immunol 2002; 169: 4098-102.

[33] Salih HR, Holdenrieder S, Steinle A. Soluble NKG2D ligands: prevalence, release, and functional impact. Front Biosci 2008; 13: 3448-56.

[34] Mincheva-Nilsson L, Nagaeva O, Chen T, et al. Placenta-derived soluble MHC class I chain-related molecules down-regulate NKG2D receptor on peripheral blood mononuclear cells during human pregnancy: a possible novel immune escape mechanism for fetal survival. J Immunol 2006; 176: 3585-92.

[35] Cerboni C, Ardolino M, Santoni A, Zingoni A. Detuning CD8+ T lymphocytes by down-regulation of the activating receptor NKG2D: role of NKG2D ligands released by activated T cells. Blood 2009; 113: 2955-64.

[36] Chitadze G, Bhat J, Lettau M, Janssen O, Kabelitz D. Generation of soluble NKG2D ligands: proteolytic cleavage, exosome secretion and functional implications. Scand J Immunol 2013; 78: 120-9.

[37] Baragano Raneros A, Suarez-Alvarez B, Lopez-Larrea C. Secretory pathways generating immunosuppressive NKG2D ligands: New targets for therapeutic intervention. Oncoimmunology 2014; 3: e28497.

[38] Visse R, Nagase H. Matrix metalloproteinases and tissue inhibitors of metalloproteinases: structure, function, and biochemistry. Circ Res 2003; 92: 827-39.

[39] Ashiru O, Boutet P, Fernandez-Messina L, et al. Natural killer cell cytotoxicity is suppressed by exposure to the human NKG2D ligand $\mathrm{MICA} * 008$ that is shed by tumor cells in exosomes. Cancer Res 2010; 70: 481-9.

[40] Fernandez-Messina L, Ashiru O, Boutet P, et al. Differential mechanisms of shedding of the glycosylphosphatidylinositol (GPI)anchored NKG2D ligands. J Biol Chem 2010; 285: 8543-51.

[41] Clayton A, Mitchell JP, Court J, Linnane S, Mason MD, Tabi Z. Human tumor-derived exosomes down-modulate NKG2D expression. J Immunol 2008; 180: 7249-58.

[42] Hedlund M, Stenqvist AC, Nagaeva O, et al. Human placenta expresses and secretes NKG2D ligands via exosomes that downmodulate the cognate receptor expression: evidence for immunosuppressive function. J Immunol 2009; 183: 340-51.

[43] Viaud S, Terme M, Flament C, et al. Dendritic cell-derived exosomes promote natural killer cell activation and proliferation: a role for NKG2D ligands and IL-15Ralpha. PLoS One 2009; 4: e4942.

[44] Cao W, Xi X, Hao Z, et al. RAET1E2, a soluble isoform of the UL16-binding protein RAET1E produced by tumor cells, inhibits NKG2D-mediated NK cytotoxicity. J Biol Chem 2007; 282: 18922-8.

[45] Eisele G, Wischhusen J, Mittelbronn M, et al. TGF-beta and metalloproteinases differentially suppress NKG2D ligand surface expression on malignant glioma cells. Brain 2006; 129: 2416-25.

[46] Salih HR, Goehlsdorf D, Steinle A. Release of MICB molecules by tumor cells: mechanism and soluble MICB in sera of cancer patients. Hum Immunol 2006; 67: 188-95.

[47] Kohga K, Takehara T, Tatsumi T, et al. Sorafenib inhibits the shedding of major histocompatibility complex class I-related chain A on hepatocellular carcinoma cells by down-regulating a disintegrin and metalloproteinase 9. Hepatology 2010; 51: 1264-73.

[48] Huang B, Sikorski R, Sampath P, Thorne SH. Modulation of NKG2D-ligand cell surface expression enhances immune cell therapy of cancer. J Immunother 2011; 34: 289-96.

[49] Chitadze G, Lettau M, Bhat J, et al. Shedding of endogenous MHC class I-related chain molecules A and B from different human tumor entities: heterogeneous involvement of the "a disintegrin and metalloproteases" 10 and 17. Int J Cancer 2013; 133: 1557-66.

[50] Groh V, Wu J, Yee C, Spies T. Tumour-derived soluble MIC ligands impair expression of NKG2D and T-cell activation. Nature 2002; 419: 734-8.

[51] Doubrovina ES, Doubrovin MM, Vider E, et al. Evasion from NK cell immunity by MHC class I chain-related molecules expressing colon adenocarcinoma. J Immunol 2003; 171: 6891-9.

[52] Song H, Kim J, Cosman D, Choi I. Soluble ULBP suppresses natural killer cell activity via down-regulating NKG2D expression. Cell Immunol 2006; 239: 22-30.

[53] Hilpert J, Grosse-Hovest L, Grunebach F, et al. Comprehensive analysis of NKG2D ligand expression and release in leukemia: implications for NKG2D-mediated NK cell responses. J Immunol 2012; 189: 1360-71.

[54] Von Lilienfeld-Toal M, Frank S, Leyendecker C, et al. Reduced immune effector cell NKG2D expression and increased levels of soluble NKG2D ligands in multiple myeloma may not be causally linked. Cancer Immunol Immunother 2010; 59: 829-39.

[55] Waldhauer I, Steinle A. Proteolytic release of soluble UL16binding protein 2 from tumor cells. Cancer Res 2006; 66: 2520-6.

[56] Groh V, Bruhl A, El-Gabalawy H, Nelson JL, Spies T. Stimulation of T cell autoreactivity by anomalous expression of NKG2D and its MIC ligands in rheumatoid arthritis. Proc Natl Acad Sci USA 2003; 100: 9452-7.

[57] Hue S, Mention JJ, Monteiro RC, et al. A direct role for NKG2D/MICA interaction in villous atrophy during celiac disease. Immunity 2004; $21: 367-77$.

[58] Molfetta R, Quatrini L, Capuano C, et al. c-Cbl regulates MICAbut not ULBP2-induced NKG2D down-modulation in human NK cells. Eur J Immunol 2014; 44: 2761-70.

[59] Montalban-Arques A, Gorkiewicz G, Mulero V, Galindo-Villegas J. Cytokine Intervention: A Double Edged Sword in the NKG2D System Regulation. Immunome Res 2014; S2: 002. Available at: http://omicsonline.com/open-access/ArchiveIMR/articleinpressimmunome-research-open-access.php

[60] Welte SA, Sinzger C, Lutz SZ, et al. Selective intracellular retention of virally induced NKG2D ligands by the human cytomegalovirus UL16 glycoprotein. Eur J Immunol 2003; 33: 194-203.

[61] Rölle A, Mousavi-Jazi M, Eriksson M, et al. Effects of human cytomegalovirus infection on ligands for the activating NKG2D re- 
ceptor of NK cells: up-regulation of UL16-binding protein (ULBP) 1 and ULBP2 is counteracted by the viral UL16 protein. J Immunol 2003; 171: 902-8.

[62] Cerboni C, Neri F, Casartelli N, et al. Human immunodeficiency virus $1 \mathrm{Nef}$ protein downmodulates the ligands of the activating receptor NKG2D and inhibits natural killer cell-mediated cytotoxicity. J Gen Virol 2007; 88: 242-50.

[63] Ebihara T, Masuda H, Akazawa T, et al. Induction of NKG2D ligands on human dendritic cells by TLR ligand stimulation and RNA virus infection. Int Immunol 2007; 19: 1145-55.

[64] Tomasec P, Wang EC, Groh V, et al. Adenovirus vector delivery stimulates natural killer cell recognition. J Gen Virol 2007; 88: 1103-8.

[65] Pappworth IY, Wang EC, Rowe M. The switch from latent to productive infection in epstein-barr virus-infected $\mathrm{B}$ cells is associated with sensitization to NK cell killing. J Virol 2007; 81: 474-82.

[66] Sene D, Levasseur F, Abel M, et al. Hepatitis C virus (HCV) evades NKG2D-dependent NK cell responses through NS5Amediated imbalance of inflammatory cytokines. PLoS Pathog 2010; 6: e1001184.

[67] Jensen H, Andresen L, Nielsen J, Christensen JP, Skov S. Vesicular stomatitis virus infection promotes immune evasion by preventing NKG2D-ligand surface expression. PLoS One 2011; 6: e23023.

[68] Sinclair A, Yarranton S, Schelcher C. DNA-damage response pathways triggered by viral replication. Expert Rev Mol Med 2006; 8: 1-11.

[69] Lilley CE, Schwartz RA, Weitzman MD. Using or abusing: viruses and the cellular DNA damage response. Trends Microbiol 2007; 15: 119-26.

[70] Turnell AS, Grand RJ. DNA viruses and the cellular DNA-damage response. J Gen Virol 2012; 93: 2076-97.

[71] Nachmani D, Stern-Ginossar N, Sarid R, Mandelboim O. Diverse herpesvirus microRNAs target the stress-induced immune ligand MICB to escape recognition by natural killer cells. Cell Host Microbe 2009; 5: 376-85.

[72] Bauman Y, Nachmani D, Vitenshtein A, et al. An identical miRNA of the human JC and BK polyoma viruses targets the stress-induced ligand ULBP3 to escape immune elimination. Cell Host Microbe 2011; 9: 93-102.

[73] Dunn C, Chalupny NJ, Sutherland CL, et al. Human cytomegalovirus glycoprotein UL16 causes intracellular sequestration of NKG2D ligands, protecting against natural killer cell cytotoxicity. $\mathrm{J}$ Exp Med 2003; 197: 1427-39.

[74] Chalupny NJ, Rein-Weston A, Dosch S, Cosman D. Downregulation of the NKG2D ligand MICA by the human cytomegalovirus glycoprotein UL142. Biochem Biophys Res Commun 2006; 346: $175-81$

[75] Ashiru O, Bennett NJ, Boyle LH, Thomas M, Trowsdale J, Wills MR. NKG2D ligand MICA is retained in the cis-Golgi apparatus by human cytomegalovirus protein UL142. J Virol 2009; 83: 12345-54.

[76] Bennett NJ, Ashiru O, Morgan FJ, et al. Intracellular sequestration of the NKG2D ligand ULBP3 by human cytomegalovirus. J Immunol 2010; 185: 1093-102.

[77] McSharry BP, Burgert HG, Owen DP, et al. Adenovirus E3/19K promotes evasion of NK cell recognition by intracellular sequestration of the NKG2D ligands major histocompatibility complex class I chain-related proteins A and B. J Virol 2008; 82: 4585-94.

[78] Schneider CL, Hudson AW. The human herpesvirus-7 (HHV-7) U21 immunoevasin subverts NK-mediated cytoxicity through modulation of MICA and MICB. PLoS Pathog 2011; 7: e1002362.

[79] Thomas M, Boname JM, Field S, et al. Down-regulation of NKG2D and NKp80 ligands by Kaposi's sarcoma-associated herpesvirus K5 protects against NK cell cytotoxicity. Proc Natl Acad Sci USA 2008; 105: 1656-61.

[80] Ward J, Bonaparte M, Sacks J, et al. HIV modulates the expression of ligands important in triggering natural killer cell cytotoxic responses on infected primary T-cell blasts. Blood 2007; 110: 120714.

[81] Fogli M, Mavilio D, Brunetta E, et al. Lysis of endogenously infected CD4+ $\mathrm{T}$ cell blasts by rIL-2 activated autologous natural killer cells from HIV-infected viremic individuals. PLoS Pathog 2008; 4: e1000101.

[82] Matusali G, Tchidjou HK, Pontrelli G, et al. Soluble ligands for the NKG2D receptor are released during HIV-1 infection and impair
NKG2D expression and cytotoxicity of NK cells. FASEB J 2013; 27: $2440-50$

[83] Nolting A, Dugast AS, Rihn S, et al. MHC class I chain-related protein A shedding in chronic HIV-1 infection is associated with profound NK cell dysfunction. Virology 2010; 406: 12-20.

[84] Andersen JL, Le Rouzic E, Planelles V. HIV-1 Vpr: mechanisms of G2 arrest and apoptosis. Exp Mol Pathol 2008; 85: 2-10.

[85] Ward J, Davis Z, DeHart J, et al. HIV-1 Vpr triggers natural killer cell-mediated lysis of infected cells through activation of the ATRmediated DNA damage response. PLoS Pathog 2009; 5: e1000613.

[86] Richard J, Sindhu S, Pham TN, Belzile JP, Cohen EA. HIV-1 Vpr up-regulates expression of ligands for the activating NKG2D receptor and promotes NK cell-mediated killing. Blood 2010; 115: 135463.

[87] Richard J, Pham TN, Ishizaka Y, Cohen EA. Viral protein R upregulates expression of ULBP2 on uninfected bystander cells during HIV-1 infection of primary CD4+ T lymphocytes. Virology 2013; 443: 248-56.

[88] Gummuluru S, Emerman M. Cell cycle- and Vpr-mediated regulation of human immunodeficiency virus type 1 expression in primary and transformed T-cell lines. J Virol 1999; 73: 5422-30.

[89] Lau A, Swinbank KM, Ahmed PS, et al. Suppression of HIV-1 infection by a small molecule inhibitor of the ATM kinase. Nat Cell Biol 2005; 7: 493-500.

[90] Norman JM, Mashiba M, McNamara LA, et al. The antiviral factor APOBEC3G enhances the recognition of HIV-infected primary $\mathrm{T}$ cells by natural killer cells. Nat Immunol 2011; 12: 975-83.

[91] Kirchhoff F. Immune evasion and counteraction of restriction factors by HIV-1 and other primate lentiviruses. Cell Host Microbe 2010; 8: 55-67.

[92] Roeth JF, Collins KL. Human immunodeficiency virus type 1 Nef: adapting to intracellular trafficking pathways. Microbiol Mol Biol Rev 2006; 70: 548-63.

[93] Fausther-Bovendo H, Sol-Foulon N, Candotti D, et al. HIV escape from natural killer cytotoxicity: nef inhibits NKp44L expression on CD4+ T cells. AIDS 2009; 23: 1077-87.

[94] Shah AH, Sowrirajan B, Davis ZB, et al. Degranulation of natural killer cells following interaction with HIV-1-infected cells is hindered by downmodulation of NTB-A by Vpu. Cell Host Microbe 2010; 8: 397-409.

[95] Matusali G, Potesta M, Santoni A, Cerboni C, Doria M. The human immunodeficiency virus type $1 \mathrm{Nef}$ and Vpu proteins downregulate the natural killer cell-activating ligand PVR. J Virol 2012; 86: 4496-504.

[96] Collins KL, Chen BK, Kalams SA, Walker BD, Baltimore D. HIV1 Nef protein protects infected primary cells against killing by cytotoxic T lymphocytes. Nature 1998; 391: 397-401.

[97] Nowbakht $\mathrm{P}$, Ionescu MC, Rohner A, et al. Ligands for natural killer cell-activating receptors are expressed upon the maturation of normal myelomonocytic cells but at low levels in acute myeloid leukemias. Blood 2005; 105: 3615-22.

[98] Mastroianni CM, Liuzzi GM. Matrix metalloproteinase dysregulation in HIV infection: implications for therapeutic strategies. Trends Mol Med 2007; 13: 449-59.

[99] Lecuroux C, Saez-Cirion A, Noel N, et al. NKG2D expression on HIV-specific CD8 $+\mathrm{T}$ cells is reduced in viremic HIV-1-infected patients but maintained in HIV controllers. J Acquir Immune Defic Syndr 2013; 62: 17-20.

[100] Shafi S, Vantourout P, Wallace G, et al. An NKG2D-mediated human lymphoid stress surveillance response with high interindividual variation. Sci Transl Med 2011; 3: 113ra124.

[101] Mavilio D, Benjamin J, Daucher M, et al. Natural killer cells in HIV-1 infection: dichotomous effects of viremia on inhibitory and activating receptors and their functional correlates. Proc Natl Acad Sci USA 2003; 100: 15011-6.

[102] Mavilio D, Lombardo G, Benjamin J, et al. Characterization of CD56-/CD16+ natural killer (NK) cells: a highly dysfunctional NK subset expanded in HIV-infected viremic individuals. Proc Natl Acad Sci USA 2005; 102: 2886-91.

[103] Conry SJ, Milkovich KA, Yonkers NL, et al. Impaired plasmacytoid dendritic cell (PDC)-NK cell activity in viremic human immunodeficiency virus infection attributable to impairments in both PDC and NK cell function. J Virol 2009; 83: 11175-87.

[104] Fu GF, Chen X, Hao S, et al. Differences in natural killer cell quantification and receptor profile expression in HIV-1 infected Chinese children. Cell Immunol 2010; 265: 37-43. 
[105] Bisio F, Bozzano F, Marras F, Di Biagio A, Moretta L, De Maria A. Successfully treated HIV-infected patients have differential expression of NK cell receptors (NKp46 and NKp30) according to AIDS status at presentation. Immunol Lett 2013; 152: 16-24.

[106] Zloza A, Kohlhapp FJ, Lyons GE, et al. NKG2D signaling on CD8(+) T cells represses T-bet and rescues CD4-unhelped CD8(+) $\mathrm{T}$ cell memory recall but not effector responses. Nat Med 2012; 18: 422-8.

[107] Chretien AS, Le Roy A, Vey N, et al. Cancer-induced alterations of NK-mediated target recognition: current and investigational Phar- macological strategies aiming at restoring NK-mediated anti-tumor activity. Front Immunol 2014; 5:122.

[108] Vanham G, Van Gulck E. Can immunotherapy be useful as a "functional cure" for infection with human immunodeficiency Virus-1? Retrovirology 2012; 9: 72.

[109] Latronico T, Liuzzi GM, Riccio P, et al. Antiretroviral therapy inhibits matrix metalloproteinase-9 from blood mononuclear cells of HIV-infected patients. AIDS 2007; 21: 677-84. 